\title{
Axial stiffness of anchoring rods embedded in elastic media
}

\author{
A. P. S. Selvadurai \\ Department of Civil Engineering, Carleton University, Ottawa, Ont., Canada KIS 5B6 \\ AND \\ R. K. N. D. RAJAPAKSE \\ Department of Civil Engineering, University of Manitoba, Winnipeg, Man., Canada R3T 2N2 \\ Received May 24, 1989 \\ Revised manuscript accepted February 7, 1990
}

\begin{abstract}
This paper presents a theoretical evaluation of the axial stiffness characteristics of elastic rods partially embedded in elastic media. The novel analytical-numerical technique is based on an energy or variational scheme in which the deformation of the bar is specified by a function, which is indeterminate to within a set of unknown constants. A minimization of the potential energy of the bar-elastic medium system is used to determine these arbitrary constants. The numerical results presented illustrate the influence of the relative flexibility of the bar, its length-to-radius aspect ratio, etc. on its axial stiffness.
\end{abstract}

Key words: anchor rods, load transfer, axial stiffness, variational methods, elastic piles, tapered bars.

\begin{abstract}
Cet article présente une évaluation théorique des caractéristiques de raideur axiale de tiges élastiques qui sont partiellement enrobées d'un matériau élastique. Cette nouvelle technique analytique-numérique repose sur une méthode variationnelle à l'intérieur de laquelle la déformation de la tige est spécifiée par une fonction, laquelle est indéterminée à l'intérieur d'un ensemble de constantes inconnues. Une minimisation de l'énergie possible du système de tiges élastiques partiellement enrobées d'un matériau élastique est utilisée pour déterminer ces constantes arbitraires. Les résultats numériques illustrent l'influence de la flexibilité relative de la tige, de son rapport longueur-rayon, etc., sur la raideur axiale.

Mots clés : tiges d'ancrage, transfert de charges, raideur axiale, méthodes variationnelles, pieux élastiques, tiges enrobées.
\end{abstract}

[Traduit par la revue]

Can. J. Civ. Eng. 17, 321-328 (1990)

\section{Introduction}

An examination of the load transfer mechanism from an elastic bar to an elastic halfspace region can be motivated by a number of useful engineering applications. The basic model of the embedded elastic bar serves as a useful approximation for the study of the axial load transfer from flexible piles to the surrounding soils, or reinforcing rods to the surrounding concrete, especially in the working load range (Fig. 1). The load transfer model is also of importance to the study of load transfer from cylindrical inclusions, which are used as reinforcement for multiphase composite materials. The fundamental work related to the load transfer from a cylindrical elastic inclusion of infinite length to an elastic medium was first examined by Muki and Sternberg (1969), who also presented approximate procedures which can be used to determine the decay of load in a bar with an arbitrary cross section. Muki and Sternberg (1970) also investigated the problem of the diffusion of axial load from an elastic bar of finite length and circular cross section which is embedded in bonded contact with an isotropic elastic halfspace. This investigation is based on the assumption that the bar behaves as a onedimensional elastic continuum which exhibits a uniform axial stress distribution. Such an assumption essentially reduces the problem to the solution of a Fredholm integral equation of the second kind. The solution procedure is valid for situations in which the length-to-diameter ratio of the bar is comparatively large. Luk and Keer (1979) presented an exact analytical formulation of the problem of a rigid cylindrical inclusion which is embedded at the surface of an isotropic elastic halfspace. A

NoTE: Written discussion of this paper is welcomed and will be received by the Editor until October 31, 1990 (address inside front cover).
Hankel transform development of the governing equations yields a system of coupled singular integral equations for the normal and shear traction distributions at the inclusionelastic medium interface.

Poulos and Davis (1968) examined the problem of the axial loading of incompressible piles embedded in elastic media by employing a discretization procedure. The interface between the pile and the elastic medium was discretized into a series of ring elements of finite length. The expressions for the axial displacement due to vertical stresses acting on these elements were derived by employing Mindlin's solution (1936) for concentrated axial load acting at the interior of the halfspace. The intensity of the interface stresses acting on each element was determined by prescribing the compatibility of vertical displacement between the pier and the elastic halfspace at the interface. The analysis neglected the influence of radial tractions acting along the interface. Suriyamongkol et al. (1973) investigated the behaviour of an axially loaded rigid cylindrical body embedded in bonded contact at the surface of an elastic halfspace. The region of the embedded body and the remainder of the halfspace were treated as a single domain and the field of distributed traction-resultants were applied at the boundary of the cylindrical region. The intensities of these traction resultants were evaluated at discrete locations by invoking the requisite axial and radial compatibility constraints. Niumpradit and Karasudhi (1979) considered the quasi-static axial load transfer from a cylindrical elastic bar into a saturated porous elastic solid. Karasudhi et al. (1984a) reconsidered the load transfer problems involving axial, lateral, and moment loading by using an improved compatibility condition proposed by Karasudhi et al. (1984b) for a torque transfer problem. Selvadurai and Rajapakse (1985) have presented a comprehensive study of the problem of load transfer 

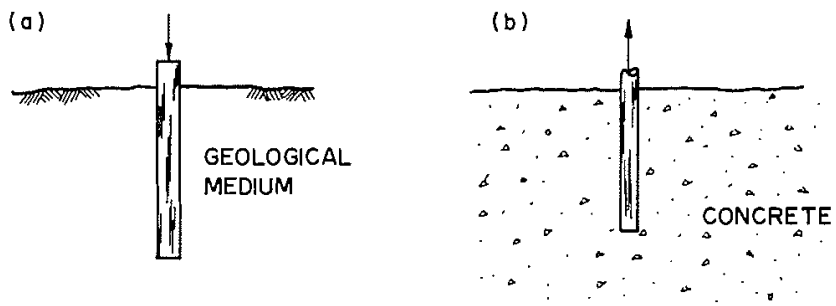

(c)
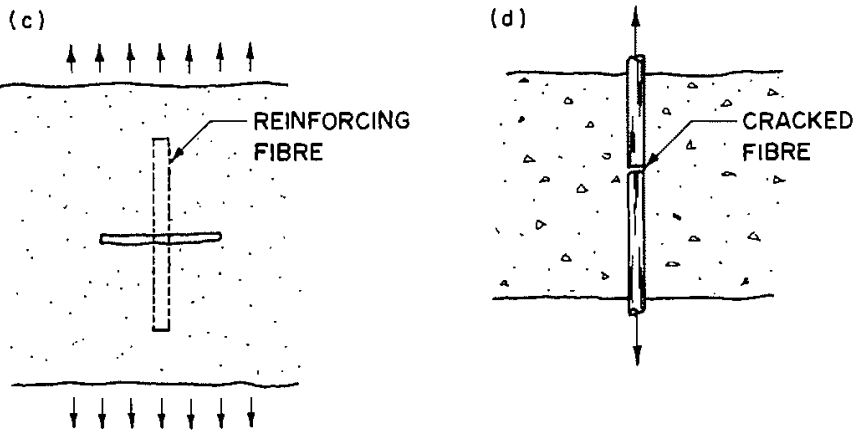

FIG. 1. Engineering situations involving load transfer from a bar to an elastic medium: ( $a$ ) axially loaded flexible pile; $(b)$ load transfer from a cylindrical bar; $(c)$ cracks in fibre reinforced solids; $(d)$ load transfer in cracked fibres.

from a hollow rigid cylindrical inclusion under general loading conditions. More recently, Selvadurai and Rajapakse (1987) have examined the problem of the torsional load transfer from an elastic bar into an isotropic elastic halfspace.

In this paper we consider the idealized problem of an axially loaded elastic bar partially embedded in an isotropic homogeneous elastic halfspace as shown in Fig. 2. It is assumed that due to the application of the axial load the bar is subjected to a deformation which includes rigid body and other terms that incorporate variations of the displacement along the length of the bar. This deformation is specified to within a set of unknown coefficients. The bar itself is assumed to behave as a one-dimensional elastic continuum. The surrounding elastic medium which is in contact with the elastic bar deforms according to the imposed deformation field. A discretized form of the stresses induced in the bar-elastic medium interface due to the imposed deformation can be computed by using a fundamental solution related to the vertical ring load acting at the interior of an elastic halfspace. Using these techniques we develop a total potential energy functional for the bar-elastic medium system. This functional includes the elastic energy of the halfspace, the elastic energy of the bar, and the potential energy of the applied axial loads. This functional is indeterminate to within the arbitrary constants characterizing the deformation of the bar. The minimization of the total potential energy functional can be used to determine these arbitrary constants. The results obtained from the present scheme are compared with those presented by Karasudhi $e t$ al. (1984a). Numerical results presented in the paper illustrate the effect of flexibility of the bar and the geometric aspect ratio on the computed axial stiffness of the bar at the surface of the halfspace region.

\section{The fundamental solution}

Figure 3 illustrates a halfspace region in which $(r, \theta, z)$ is the cylindrical polar coordinate system, and the associated rec-

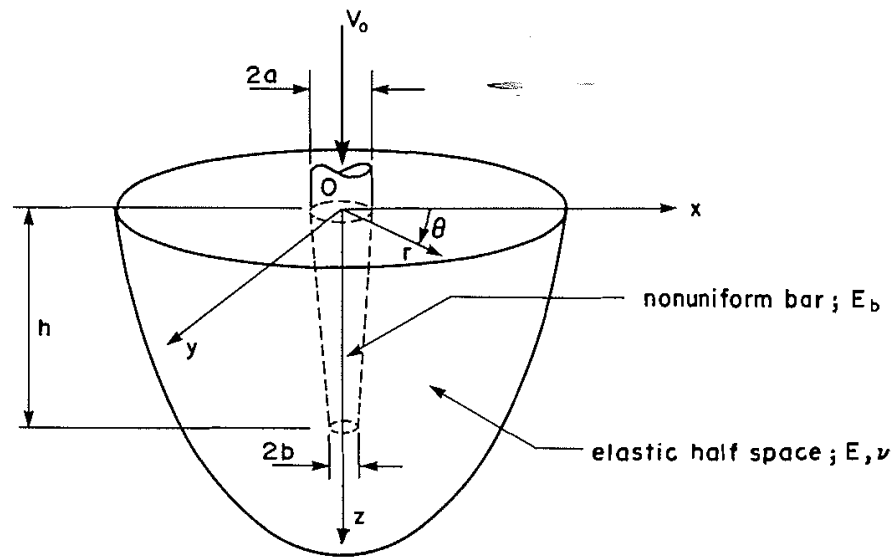

Fig. 2. Geometry of the nonuniform bar embedded in an elastic medium.

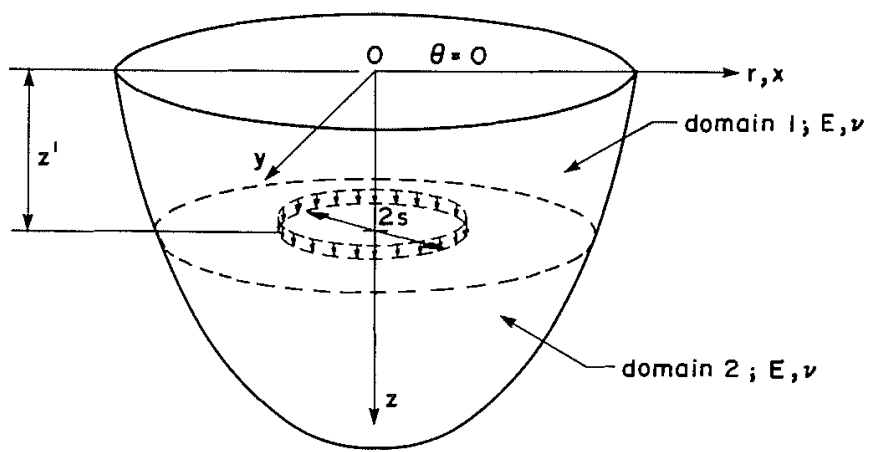

FlG. 3. System considered in the derivation of the fundamental solution.

tangular Cartesian coordinate system $(x, y, z)$ is chosen such that the $z$-axis is normal to the free surface. For an isotropic linear elastic medium undergoing torsion-free axisymmetric deformations, the displacement equations of equilibrium in a medium which is void of body forces take the form

[1]

$$
\nabla^{2} u+\frac{1}{1-2 \nu} \frac{\partial \Delta}{\partial r}-\frac{u}{r^{2}}=0
$$

$$
\nabla^{2} w+\frac{1}{1-2 \nu} \frac{\partial \Delta}{\partial z}=0
$$

where $u$ and $w$ are the components of the displacement vector referred to the cylindrical polar coordinates $r$ and $z$, respectively; $\nu$ is Poisson's ratio; $\nabla^{2}$ and $\Delta$ are the Laplacian operator and dilatation, respectively, defined by

[2]

$$
\nabla^{2}=\frac{\partial^{2}}{\partial r^{2}}+\frac{1}{r} \frac{\partial}{\partial r}+\frac{\overline{\partial^{2}}}{\partial z^{2}}
$$

$$
\Delta=\frac{\partial u}{\partial r}+\frac{u}{r}+\frac{\partial w}{\partial z}
$$

Muki (1960) presented a Fourier (in terms of $\theta$ ) - Hankel (in terms of $r$ ) representation of the general solution of [1] by expressing the displacements in terms of a combination of biharmonic and harmonic functions. Accordingly, the displacements $u$ and $w$ can be expressed as 


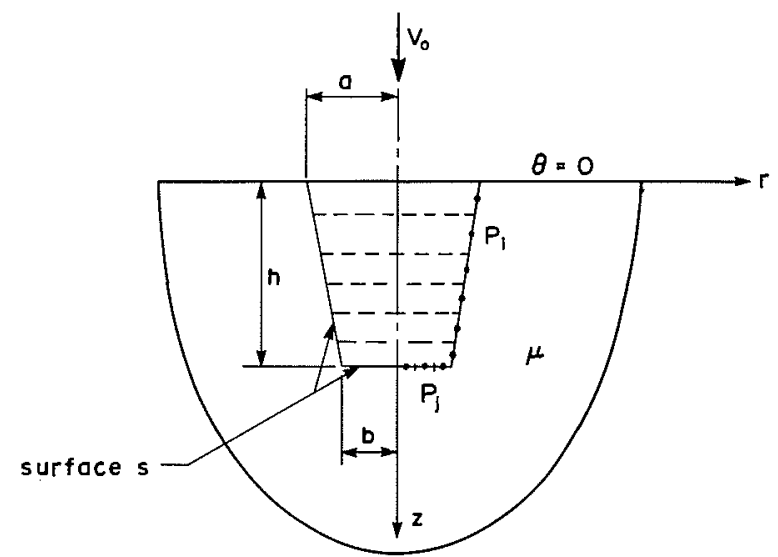

Fig. 4. Discretization for the fictitious contact surface $S$.

[3]

$$
u(r, z)=\int_{0}^{\infty} \frac{\mathrm{d}}{\mathrm{d} z} G(\xi, z) \xi^{2} J_{1}(\xi r) \mathrm{d} \xi
$$

$$
\begin{aligned}
w(r, z)=\int_{0}^{\infty}\left[(1-2 \nu) \frac{\mathrm{d}^{2}}{\mathrm{~d} z^{2}} G(\xi, z)\right. \\
\left.-2(1-\nu) \xi^{2} G(\xi, z)\right] \xi J_{0}(\xi r) \mathrm{d} \xi
\end{aligned}
$$

where

$$
G(\xi, z)=(A+B z) \mathrm{e}^{-\xi z}+(C+D z) \mathrm{e}^{\xi z}
$$

In [3], $J_{m}$ is the Bessel function of the first kind of $m$ th order and $A(\xi), B(\xi), C(\xi)$, and $D(\xi)$ are arbitrary functions which should be determined by invoking appropriate boundary and (or) continuity conditions. At this stage, it is convenient to nondimensionalize the problem by defining a length parameter, $a$, which denotes the radius of the cylindrical bar at $z=$ 0 as the unit of length.

In the ensuing section dealing with variational formulation, we need to obtain the traction distribution generated along a fictitious contact surface $S$ (Fig. 4) on the extended halfspace (i.e., uniform halfspace without an inclusion) due to a set of deformations imposed along $S$. To the authors' knowledge, an exact analytical solution does not exist for the traction distribution generated due to an arbitrary displacement pattern imposed along $S$. The most efficient and accurate way to compute the traction distribution along $S$ is to discretize the surface $S$ and to develop a numerical solution. The surface $S$ is discretized into ring elements as shown in Fig. 4. It may be observed that any contact surface corresponding to a nonuniform bar can be discretized by the three different types of elements referred to the surface $S$, namely, vertical (VE), inclined (IE), and base (BE) elements shown in Fig. 5. The traction distribution within each element is as shown in Fig. 5.

Since we are concerned with the evaluation of the axial stiffness of long elastic bars, it is reasonable to neglect the displacement and tractions in radial directions in determining the traction, $T(r, z)$, in the $z$-direction on the surface $S$ due to imposed displacement field $w(r, z)$. To obtain a numerical solution for $T(r, z)$ at discrete points along the surface $S$, we need to derive the fundamental solution corresponding to a concentrated circular ring load acting in the $z$-direction at the interior of a halfspace as shown in Fig. 3. Referring to Fig. 3, and by defining a fictitious plane at $z=z^{\prime}$, we can reduce the problem to one that has two domains. The superscript (or sub-

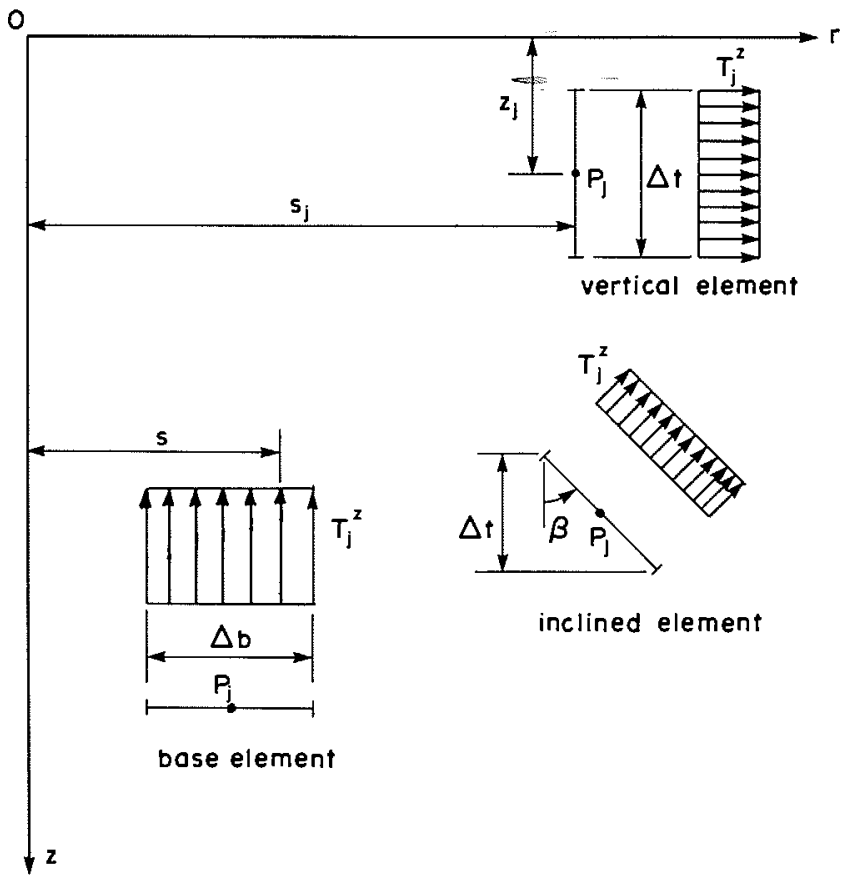

Frg. 5. Basic elements used to model the load transfer mechanism at the fictitious contact surface $S$.

script) $i(i=1,2)$ is used to identify the quantities associated with the appropriate domain. The displacement field in each domain has the general form given by [3] consisting of functions $A_{i}(\xi), B_{i}(\xi), C_{i}(\xi)$, and $D_{i}(\xi)$. In the domain 2 , however, to ensure regularity of displacements and stresses derived from [3], the terms $C_{2}(\xi)$ and $D_{2}(\xi)$ are set equal to zero. The remaining six functions $A_{1}(\xi), B_{1}(\xi), C_{1}(\xi), D_{1}(\xi), A_{2}(\xi)$, and $B_{2}(\xi)$ are determined from the following boundary and continuity conditions:

$$
\text { [4] } \begin{aligned}
w^{(1)}\left(r, z^{\prime}\right)=w^{(2)}\left(r, z^{\prime}\right) & \\
\sigma_{z z}^{(1)}\left(r, z^{\prime}\right)-\sigma_{z z}^{(2)}\left(r, z^{\prime}\right) & =\delta(r-s) \\
& =\int_{0}^{\infty} s \xi J_{0}(\xi s) J_{0}(\xi r) \mathrm{d} \xi \\
\sigma_{z r}^{(1)}\left(r, z^{\prime}\right)-\sigma_{z r}^{(2)}\left(r, z^{\prime}\right) & =0
\end{aligned}
$$

The solution of the set of simultaneous equations corresponding to [4] results in expressions for displacements of the halfspace. It is found that the expression for vertical displacement takes the form

$$
\text { [5] } \begin{aligned}
w\left(r, z ; s, z^{\prime}\right)= & \frac{s}{8 \mu(1-\nu)} \int_{0}^{\infty}\left\{\left[\lambda_{1}+\xi\left|z-z^{\prime}\right|\right]\right. \\
& \times \mathrm{e}^{-\xi\left|z-z^{\prime}\right|}+\left[\lambda_{2}+\lambda_{1} \xi\left(z+z^{\prime}\right)+2 \xi^{2} z z^{\prime}\right] \\
& \left.\times \mathrm{e}^{-\xi\left(z+z^{\prime}\right)}\right\} J_{0}(\xi s) J_{0}(\xi r) \mathrm{d} \xi \\
& \quad 0 \leq z, z^{\prime}<\infty
\end{aligned}
$$

where $\lambda_{1}=3-4 \nu$ and $\lambda_{2}=8 \nu^{2}-12 \nu+5$.

The displacement, $f_{i j}$, at point $P_{i}\left(r_{i}, z_{i}\right)$ on the interface $S$ 
due to tractions of unit intensity distributed over any type of element $(j)$ shown in Fig. 4 can be derived by integrating the fundamental solution given by [5] across the width of the element. It is noted that the fundamental solution could be integrated analytically for tractions applied over the vertical and base elements. For the inclined element, however, a numerical integration has to be employed across the element width to compute $f_{i j}$. The expression for $f_{i j}$ corresponding to each type of element depicted in Fig. 5 is given below.

For a vertical element,

$$
\begin{aligned}
f_{i j}= & \frac{s_{j}}{8 \mu(1-\theta)}\left\{\int _ { z ^ { \prime } = z _ { j } - \Delta t / 2 } ^ { z ^ { \prime } = z _ { j } + \Delta t / 2 } \left[\left(\lambda_{1}+1\right) I_{1}(0,0 ; 0)\right.\right. \\
& \left.+\left(\lambda_{2}+\lambda_{1}\right) I_{2}(0,0 ; 0)\right] \mathrm{d} z^{\prime}+\left[\left(z_{i}-z^{\prime}\right) I_{1}(0,0 ; 0)\right. \\
& -\lambda_{1}\left(z_{i}+z^{\prime}\right) I_{2}(0,0 ; 0)-2 z_{i} z^{\prime} I_{2}(0,0 ; 1) \\
& \left.\left.-2 z_{i} I_{2}(0,0 ; 0)\right]_{z^{\prime}=z_{j}-\Delta t / 2}^{z^{\prime}=z_{j}+\Delta t / 2}\right\}
\end{aligned}
$$

For a base element,

$$
\begin{aligned}
\text { [7] } f_{i j}= & \frac{1}{8 \mu(1-\nu)}\left[s \left\{\lambda_{1} I_{1}^{*}(0,1 ;-1)+\left(z_{j}-z_{i}\right) I_{1}^{*}(0,1 ; 0)\right.\right. \\
& +\lambda_{2} I_{2}^{*}(0,1 ;-1)+\lambda_{1}\left(z_{i}+z_{j}\right) I_{2}^{*}(0,1 ; 0) \\
& \left.\left.+2 z_{i} z_{j} I_{2}^{*}(0,1 ; 1)\right\}\right]_{s=s_{j}-\Delta b / 2}^{s=s_{j}+\Delta b / 2}
\end{aligned}
$$

In [6] and [7],

[8a] $I_{1}(m, n ; p)=\int_{0}^{\infty} J_{m}\left(\xi r_{i}\right) J_{n}\left(\xi s_{j}\right) \xi^{p} \mathrm{e}^{-\xi\left|z_{i}-z^{\prime}\right|} \mathrm{d} \xi$

$$
I_{2}(m, n ; p)=\int_{0}^{\infty} J_{m}\left(\xi r_{i}\right) J_{n}\left(\xi s_{j}\right) \xi^{p} \mathrm{e}^{-\xi\left(z_{i}+z^{\prime}\right)} \mathrm{d} \xi
$$

[8c] $\quad I_{1}^{*}(m, n ; p)=\int_{0}^{\infty} J_{m}\left(\xi r_{i}\right) J_{n}(\xi s) \xi^{p} \mathrm{e}^{-\xi\left(z_{j}-z_{i}\right)} \mathrm{d} \xi$

[8d] $I_{2}^{*}(m, n ; p)=\int_{0}^{\infty} J_{m}\left(\xi r_{i}\right) J_{n}(\xi s) \xi^{p} \mathrm{e}^{-\xi\left(z_{j}+z_{i}\right)} \mathrm{d} \xi$

For an inclined element the displacement expression given by [5] is numerically integrated over the thickness of the element by using Simpson's rule.

\section{The variational formulation}

Consider an elastic halfspace with an embedded elastic bar as shown in Fig. 1. It is assumed that the bar is subjected to an axial load, $V_{0}$, at $z=0$ and that the bar is perfectly bonded to the surrounding elastic medium along its contact surface. Since we are concerned with the deformation of an elastic bar which has a large length-to-radius ratio, it is well justified (see, e.g., Muki and Sternberg 1969, 1970) to assume onedimensional behaviour for the embedded bar. The state of deformation represented by the one-dimensional theory can be expressed in the form

[9] $\quad w(z)=\sum_{n=1}^{N} \alpha_{n} \mathrm{e}^{-(n-1) z / h}$

The above displacement profile along the bar is indeterminate to within the arbitrary coefficients $\alpha_{1}, \alpha_{2}, \ldots, \alpha_{N}$.

Considering the displacement field defined by [9], the strain energy, $U_{b}$, of a tapered elastic bar as shown in Fig. 5 can be expressed as
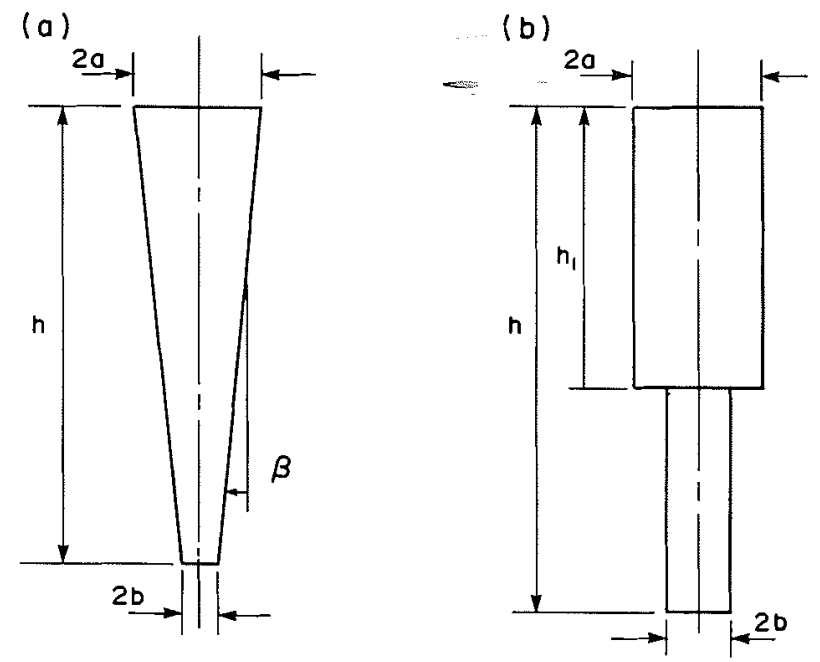

F1G. 6. Geometry of nonuniform bars considered in the present study. (a) uniform tapering; $(b)$ step tapering.

$$
U_{\mathrm{b}}=\sum_{n=1}^{N} \sum_{m=1}^{M} D_{m n} \alpha_{n} \alpha_{m}
$$

where

$$
\text { [11a] } \begin{aligned}
D_{m n}= & \frac{\pi E^{*}(m-1)(n-1)}{2 h^{2}}\left\{\frac{h a^{2}\left(1-\mathrm{e}^{-(m+n-2)}\right)}{(m+n-2)}\right. \\
& +\frac{2 h^{2} a \tan \beta \mathrm{e}^{-(m+n-2)}}{(m+n-2)} \\
& -\frac{2 h^{2} a \tan \beta\left(1-\mathrm{e}^{-(m+n-2)}\right)}{(m+n-2)^{2}} \\
& -\tan ^{2} \beta \mathrm{e}^{-(m+n-2)}\left[\frac{h^{3}}{(m+n-2)}+\frac{2 h^{3}}{(m+n-2)^{2}}\right] \\
& \left.+\frac{2 \tan ^{2} \beta h^{3}}{(m+n-2)^{3}}\left(1-\mathrm{e}^{-(m+n-2)}\right)\right\},
\end{aligned}
$$

$$
m+n \neq 2
$$

$[11 b] \quad D_{11}=0$

[11c] $E^{*}=E_{\mathrm{b}}-E$

[11d] $\tan \beta=(a-b) / h$

Note that by virtue of the system to be considered in deriving the tractions due to deformation specified by [9], the modulus of elasticity of the bar has to be modified as given by [11c]. For a step-tapered bar as shown in Fig. 6, the strain energy, $U_{\mathrm{b}}$, can also be expressed in the form of [10], except that the relevant expression for $D_{m n}$ takes the form

$$
\begin{aligned}
D_{m n}=\frac{\pi E^{*}(n-1)(m-1)}{2 h} & {\left[a^{2}-a^{2} \mathrm{e}^{-(m+n-2) h_{1} / h}\right.} \\
& \left.+b^{2} \mathrm{e}^{-(m+n-2) h_{1} / h}-a^{2} \mathrm{e}^{-(m+n-2)}\right]
\end{aligned}
$$

The strain energy of the extended elastic medium, due to the deformation imposed along the contact surface $S$, could be computed if the tractions acting on this surface are known. These tractions are computed by imposing independently the deformation corresponding to each term of [9] with $\alpha_{1}, \alpha_{2}$, $\ldots, \alpha_{n}$ equal to unity and then formulating the flexibility 
TABLE 1. Convergence of results for different values of $N$ and $N_{t}$

\begin{tabular}{ccc}
\hline \hline & \multicolumn{2}{c}{$\Delta_{0} E A / V_{0} a$} \\
\cline { 2 - 3 }$N$ & $N_{t}=12$ & $N_{t}=22$ \\
\hline 2 & 0.5821 & 0.5830 \\
4 & 0.6041 & 0.6034 \\
6 & 0.6054 & 0.6041 \\
8 & 0.6057 & 0.6043 \\
10 & 0.6058 & 0.6043 \\
\hline Note: $h / a=10.0 ; \nu=0.25 ; E_{\mathrm{b}} / E=10.0 ; b / a=1.0$
\end{tabular}

equation for the contact surface $S^{*}$. Let us denote the traction in the $z$-direction generated on the $j$ th element due to the $n$th term of [9] with $\alpha_{n}=1$ as $T_{n j}$. Using the influence function $f_{i j}$ defined previously, we can write the following flexibility equation to determine $T_{n j}$.

$$
\begin{aligned}
& \text { [13] }\left[f_{i j}\right]\left\{T_{n j}\right\}=\left\{w_{n i}\right\}, \quad i, j=1, \ldots, N_{t} \\
& n=1, \ldots, N
\end{aligned}
$$

where

$$
\begin{aligned}
& {[14 a]\left\{T_{n j}\right\}=<T_{n 1}, \ldots, T_{n i}, \ldots, T_{n N_{t}}>^{\mathrm{T}}} \\
& {[14 b]\left\{w_{n i}\right\}=<\beta_{n 1}, \ldots, \beta_{n i}, \ldots, \beta_{n N_{t}}>^{\mathrm{T}}}
\end{aligned}
$$

and

[14c] $\beta_{n i}=\mathrm{e}^{-(n-1) z_{i} / h}$

The solution of the system of simultaneous equations results in $\left\{T_{n j}\right\}$ for $n=1, \ldots, N$. The tractions acting on the $j$ th element at the bar-halfspace interface is denoted by $\bar{T}_{z j}$ which can be expressed in the form

$$
\text { [15] } \quad \bar{T}_{z j}=\sum_{n=1}^{N} \alpha_{n} T_{n j}
$$

expressed as

$$
\text { [16] } U_{\mathrm{HS}}=\frac{1}{2} \int_{S} \bar{T}(r, z) w(r, z) \mathrm{d} S
$$

where $S$ denotes the fictitious contact surface on the extended elastic medium; $\bar{T}(r, z)$ is the continuous form of the traction acting on the contact surface which is expressed in the discrete form as given by [15]. Considering [9] and [15], [16] can be expressed as

[17] $\quad U_{\mathrm{HS}}=\pi \sum_{n=1}^{N} \sum_{m=1}^{N} \sum_{j=1}^{N_{f}} \alpha_{n} \alpha_{m} T_{n j} r_{j} \mathrm{e}^{-(m-1) z_{j} / h} \Delta t_{j}$

where $\Delta t_{j}=\Delta z / \cos \beta$ if $j$ th element is a VE or IE and $\Delta t_{j}=$ $\Delta r$ if $j$ th element is a BE.

The total potential energy functional of the system, $\bar{U}$, can be written as

$$
\text { [18] } \bar{U}=U_{\mathrm{b}}+U_{\mathrm{HS}}-V_{0} \sum_{n=1}^{N} \alpha_{n}
$$

\begin{tabular}{|c|c|}
\hline \multicolumn{2}{|c|}{ (a) $h / a=10.0 ; \nu=0.25$} \\
\hline 0.7896 & 0.7729 \\
\hline 0.6256 & 0.6043 \\
\hline 0.4019 & 0.3904 \\
\hline 0.3618 & 0.3431 \\
\hline 0.3266 & 0.3182 \\
\hline 0.3220 & 0.3137 \\
\hline 0.3167 & 0.3096 \\
\hline
\end{tabular}

By minimizing the total potential energy functional (i.e., $\left.\partial \bar{U} / \partial \alpha_{i}=0, i=1,2, \ldots, N\right)$, the following system of linear simultaneous equations can be obtained:
TABLE 2. Comparison of nondimensional axial displacement of a uniform elastic bar

\begin{tabular}{|c|c|c|c|c|}
\hline \multirow[b]{2}{*}{$h / a$} & \multicolumn{4}{|c|}{$\Delta_{0} E A / V_{0} a$} \\
\hline & $\begin{array}{c}b / a=1.0 \\
\text { uniform }\end{array}$ & $\begin{array}{l}\qquad b / a=0.5 \\
\text { uniform taper }\end{array}$ & $\begin{array}{l}b / a=0.25 \\
\text { uniform taper }\end{array}$ & $\begin{array}{c}b / a=0.5 \\
h_{1} / h=0.5 \\
\text { step taper }\end{array}$ \\
\hline \multicolumn{5}{|c|}{ (a) $E_{\mathrm{b}} / E=10.0 ; \nu=0.25$} \\
\hline 5 & 0.6812 & 0.7426 & 0.7896 & 0.7153 \\
\hline 10 & 0.6043 & 0.6630 & 0.6945 & 0.6332 \\
\hline 15 & 0.5936 & 0.6376 & 0.6604 & 0.6085 \\
\hline 20 & 0.5906 & 0.6252 & 0.6429 & 0.5986 \\
\hline 25 & 0.5895 & 0.6179 & 0.6322 & 0.5945 \\
\hline 30 & 0.5893 & 0.6134 & 0.6255 & 0.5927 \\
\hline \multicolumn{5}{|c|}{ (b) $E_{\mathrm{b}} / E=10^{5} ; \nu=0.25$} \\
\hline 5 & 0.4995 & 0.5446 & 0.5986 & 0.5360 \\
\hline 10 & 0.3096 & 0.3562 & 0.3887 & 0.3535 \\
\hline 15 & 0.2385 & 0.2709 & 0.2935 & 0.2696 \\
\hline 20 & 0.1966 & 0.2210 & 0.2382 & 0.2205 \\
\hline 25 & 0.1684 & 0.1880 & 0.2018 & 0.1876 \\
\hline 30 & 0.1479 & 0.1642 & 0.1757 & 0.1640 \\
\hline
\end{tabular}

\begin{tabular}{c}
$\Delta_{0} E A / V_{0} a$ \\
\cline { 2 - 3 }$E_{\mathrm{b}} / E$
\end{tabular}

(b) $h / a=5.0 ; \nu=0.25$

$\begin{array}{ll}0.8261 & 0.8140 \\ 0.6893 & 0.6812 \\ 0.5285 & 0.5457 \\ 0.5049 & 0.5232 \\ 0.4850 & 0.5043 \\ 0.4820 & 0.5019 \\ 0.4810 & 0.4995\end{array}$

TABLE 3. Comparison of nondimensional axial displacement for bars of different geometry

$$
\text { [19] } \begin{aligned}
\sum_{n=1}^{N} \alpha_{n}\left\{2 D_{n i}+\pi \sum_{j=1}^{N_{1}}\left[r _ { j } \left(T_{n j} \mathrm{e}^{-(i-1) z_{j} / h}\right.\right.\right. \\
\left.\left.+T_{i j} \mathrm{e}^{\left.-(n-1) z_{j} / h\right) \bar{\Delta} t_{j}}\right]\right\}=V_{0}, \quad i=1, \ldots, N
\end{aligned}
$$

The solution of the system of simultaneous equations given by [19] gives the numerical values of the arbitrary constants $\alpha_{1}, \ldots, \alpha_{N}$. The displacements of the bar and the fictitious traction acting on $S$ due to the applied force $V_{0}$ could be evaluated by using [9]-[15].

For the case of an infinitely rigid bar, $\alpha_{2}, \ldots, \alpha_{N}=0$; and [19] reduces to

[20] $\quad \alpha, \sum_{j=1}^{N_{1}}\left[2 \pi r_{j} T_{! j}^{z} \Delta t_{j}\right]=V_{0}$ 

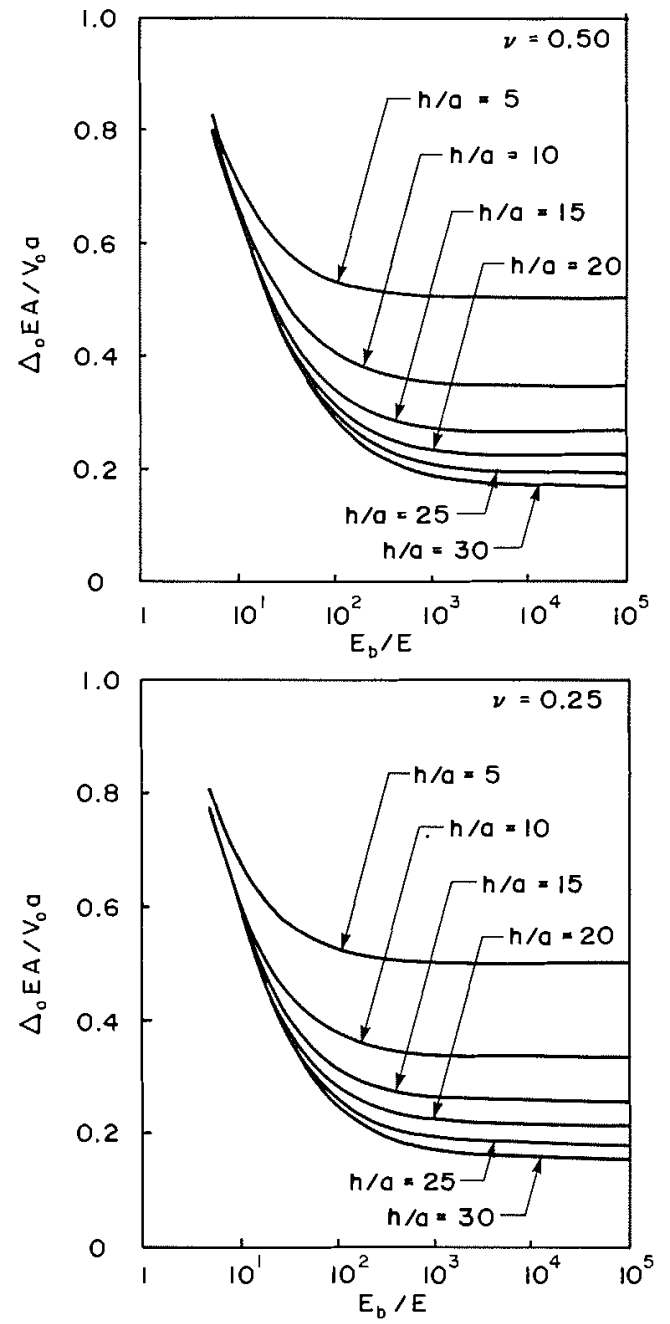

FIG. 7. Axial displacement of uniform bars: $b / a=1.0$.

It may be noted that if we consider a rigid bar and solve [13] for the appropriate rigid body displacement mode $(n=1$ only), then [20] is simply obtained by the consideration of global equilibrium of the bar.

\section{Numerical solution scheme}

The first step of the numerical solution is to solve the system of simultaneous equations given by [13] to obtain $\left\{T_{n j}\right\}$ for different $n$. This involves the computation of the influence function, $f_{i j}$, for each element. It may be noted that the influence function, $f_{i j}$, consists of several integrals of the Lipschitz-Hankel type, defined by

\section{[21] $I(p, q ; \lambda)=\int_{0}^{\infty} J_{p}(\xi r) J_{q}(\xi s) \mathrm{e}^{-\eta \xi \xi \lambda} \mathrm{d} \xi$}

Numerical evaluation of such integrals are discussed by Eason et al. (1955) and Selvadurai and Rajapakse (1985). Once the numerical solution of [13] is obtained, the system of simultaneous equations given by [19] is solved to obtain the numerical values of $\alpha_{n}$. Based on the solution procedure described in the preceding sections, the authors have developed a computer code to evaluate the axial stiffness of elastic bars. The input parameters include Young's modulus of the bar, the shear modulus, and Poisson's ratio of the elastic halfspace, the geometry of the bar, and the values of $N_{t}$ and $N$.
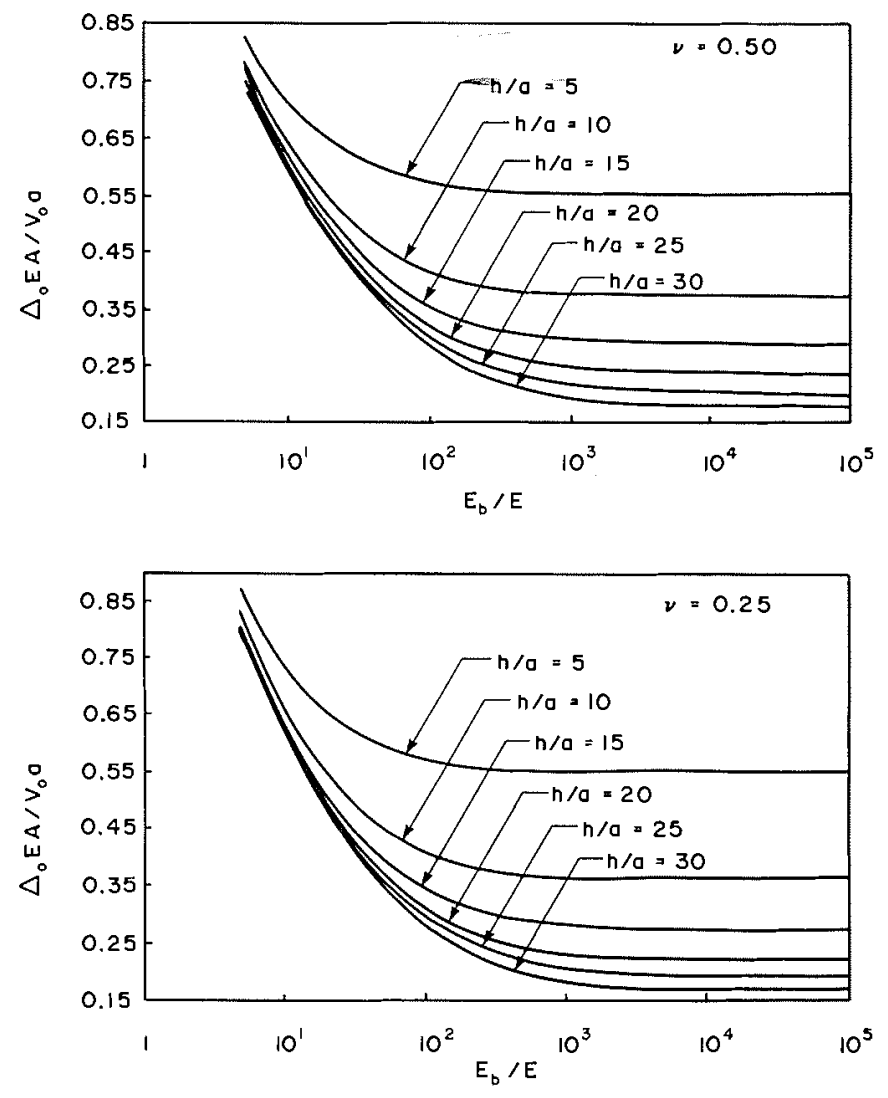

FIG. 8. Axial displacement of uniformly tapered bars: $b / a=0.50$.
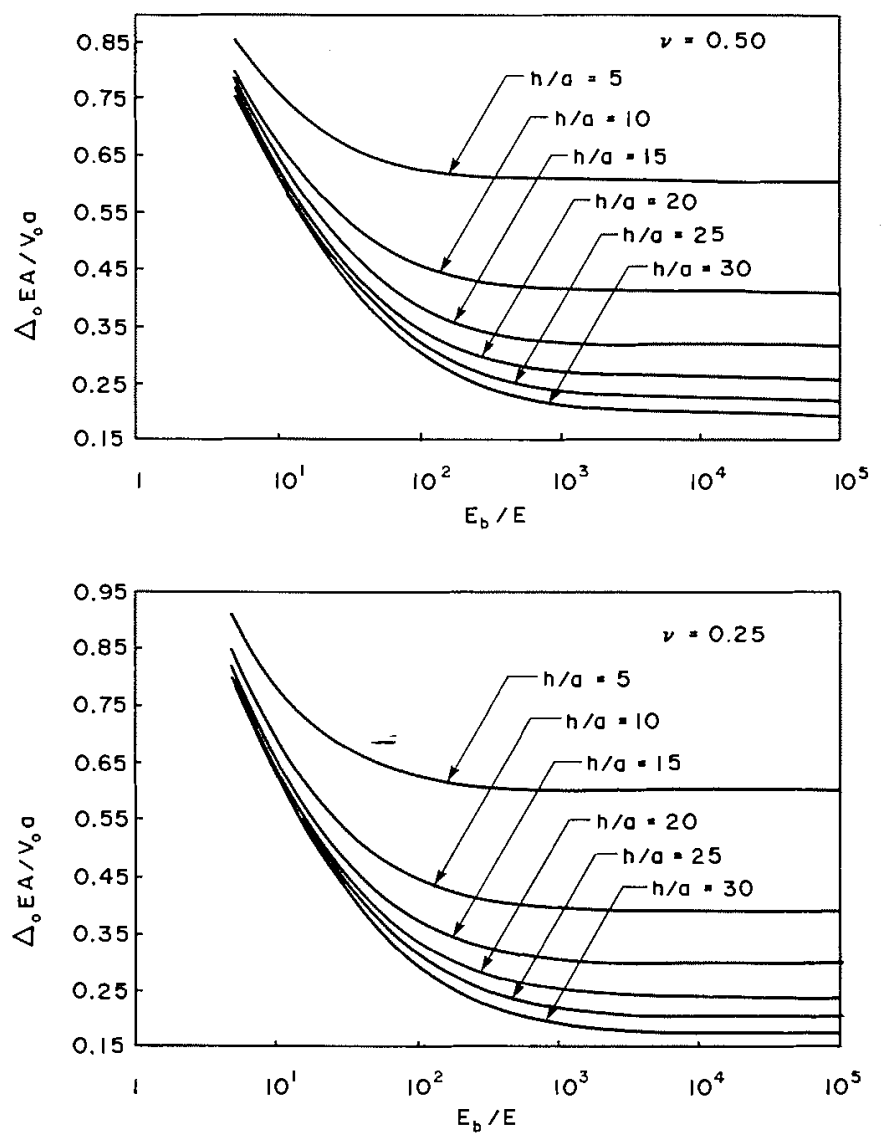

FiG. 9. Axial displacement of uniformly tapered bars: $b / a=0.25$. 

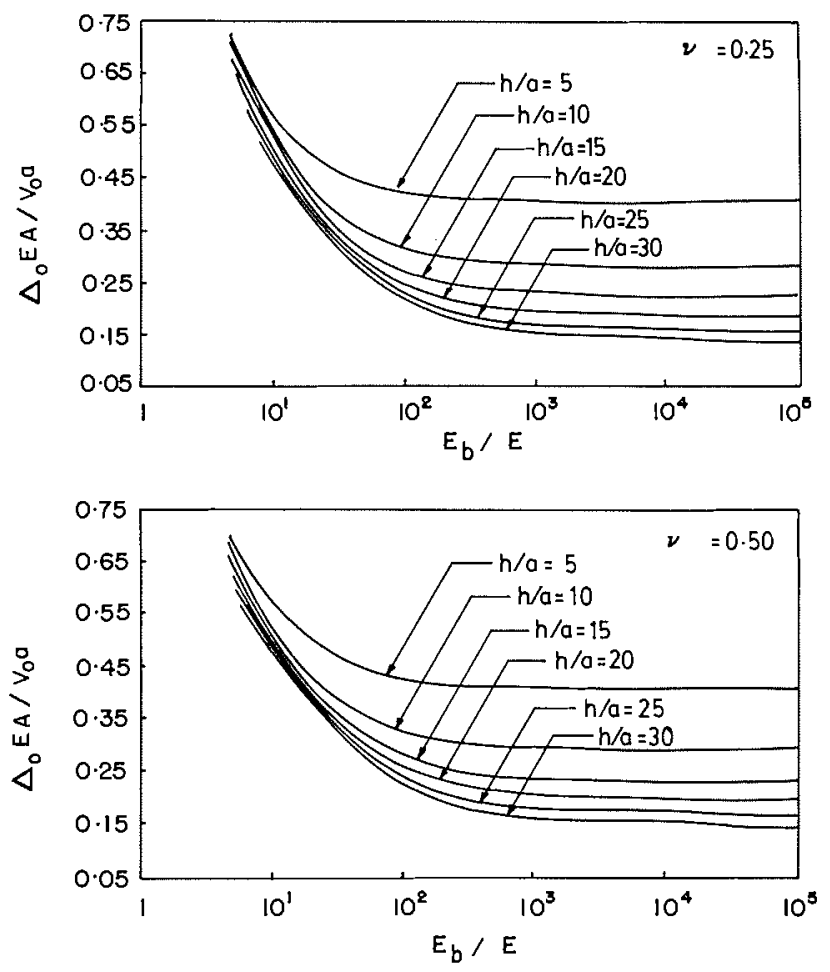

FIG. 10. Axial displacement of uniformly tapered bars: $b / a=1.50$.
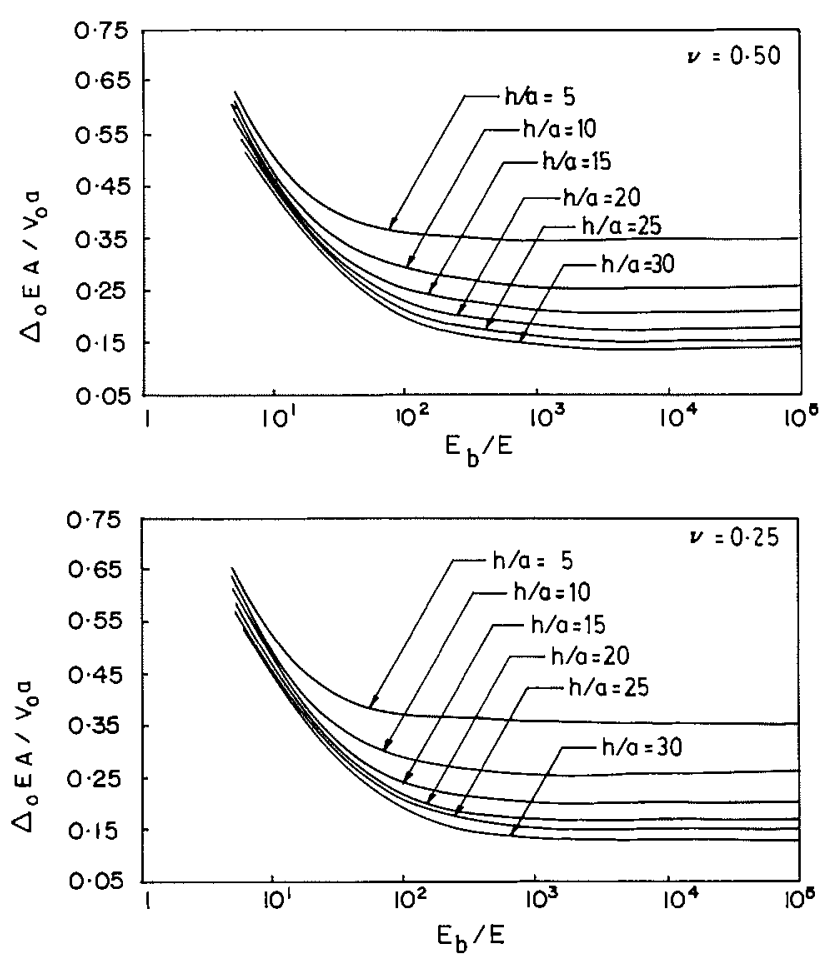

FiG. 11. Axial displacement of uniformly tapered bars: $b / a=2.0$.

The program computes displacement and tractions in the $z$ direction at discrete points on the contact surface and the load transfer curves.

\section{Discussion of results and conclusions}

The first step of the parametric study is to investigate the
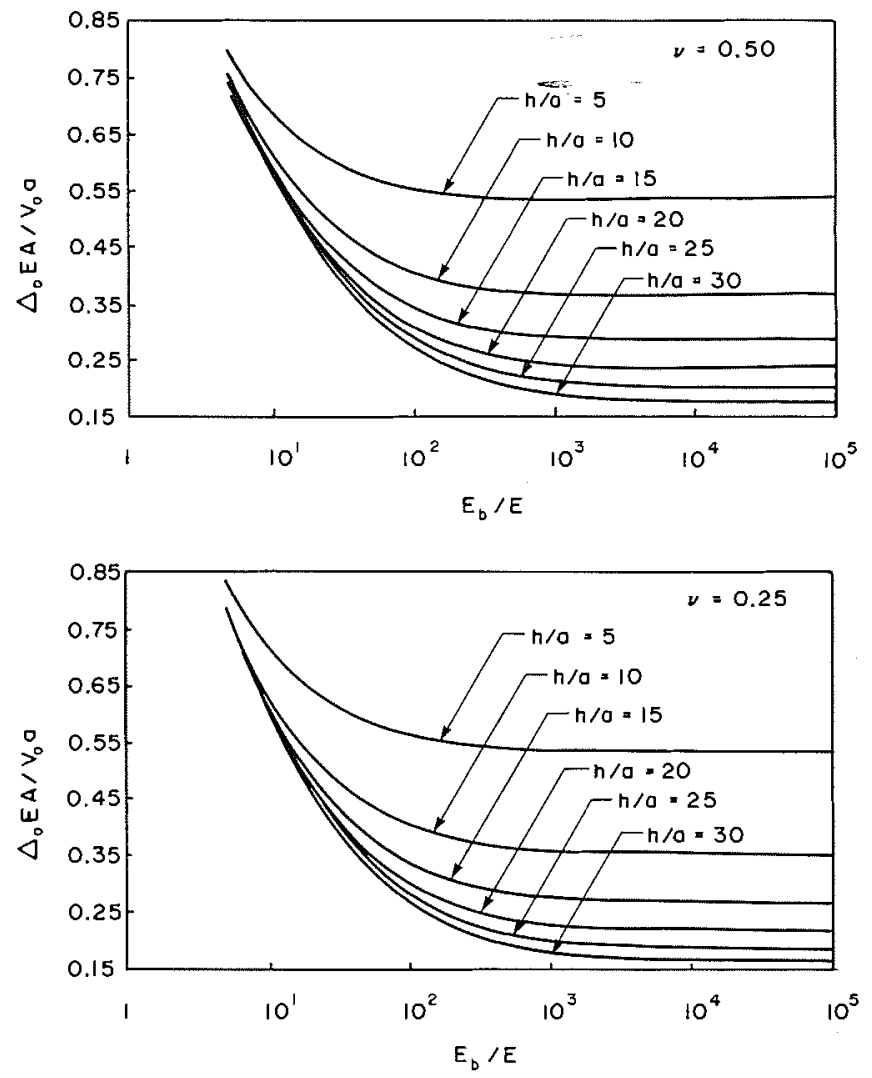

Frg. 12. Axial displacement of step tapered bars: $b / a=0.50$; $h_{1} / h=0.5$.

convergence of solutions with respect to the number of terms $(N)$ considered in the assumed displacement approximation given by [9] and the total number of elements $\left(N_{t}\right)$ used in the discretization of the contact surface $S$. Table 1 presents the nondimensionalized axial displacement, $\triangle_{0} E A / V_{0} a\left(\Delta_{0}\right.$ is the axial displacement of the bar at $z=0$ due to the axial load $V_{0}$, $A$ is the cross-sectional area of the bar at $z=0$ ), for a uniform elastic bar $\left(h / a=10.0, E_{\mathrm{b}} / E=10.0, \nu=0.25\right)$ embedded in an elastic halfspace. It is evident that the solution shows good convergence with respect to both $N$ and $N_{t}$. Table 2 presents the comparison of the nondimensionalized axial displacement for a uniform elastic bar obtained with the current procedure with that obtained by Karasudhi et al. (1984a). The numerical results agree very closely, the difference being less than $5 \%$. This slight difference may be due to the fact that in the results given by Karasudhi et al. (1984a), the LipschitzHankel integrals are evaluated by means of a numerical integration scheme, whereas in the present study we express these integrals in terms of complete elliptic integrals (see, e.g., Eason et al. 1955) which are accurately computed by a highprecision software library for special mathematical functions. Table 3 presents a comparison of the nondimensionalized axial displacement for bars having different geometries. As can be seen from the table, the effect of nonuniformity is more pronounced for shorter bars than for longer bars, with similar conclusions derived for bars with higher $E_{\mathrm{b}} / E$ ratios. Figures 7-12 present the nondimensionalized axial displacement of uniform, nonuniform, and step-tapered bars having length-toradius ratios in the range 5-30 and $E_{\mathrm{b}} / E$ ratios in the range $5-10^{5}$. These figures show that when the bar is flexible $\left(E_{\mathrm{p}}\right.$ is low) the stiffness tends to be independent of length. The 
change in Poisson's ratio of the halfspace from 0.25 to 0.50 is found to change the axial displacement by less than $3 \%$ for flexible bars.

Finally, it can be concluded that the variational scheme presented in this study is an efficient and accurate procedure for solving axial load transfer problems involving flexible elastic bars. This method can be extended to analyse general load transfer problems without any fundamental difficulty. The numerical results presented in the paper, although limited in scope, illustrate the significant influence of the relative elastic properties of the bar-elastic medium system on the axial stiffness of the bar at the surface level.

Eason, G., Noble, B., and Sneddon, I. N. 1955. On certain integrals of the Lipschitz-Hankel type involving products of Bessel functions. Philosophical Transactions of the Royal Society, A247: $529-551$.

Karasudhi, P., Rajapakse, R. K. N. D., and Liyanage, K. K. 1984a. A reconsideration of elastostatic load transfer problems involving a halfspace. Transactions of the Canadian Society for Mechanical Engineering, 8: 219-226.

Karasudhi, P., Raja pakse, R. K. N. D., and Hwang, B. Y. $1984 b$. Torsion of a long cylindrical elastic bar partially embedded in a layered halfspace. International Journal of Solids and Structures, 20: $1-11$.

LuK, V. K., and KeER, L. M. 1979. Stress analysis for an elastic halfspace containing an axially loaded rigid cylindrical rod. International Journal of Solids and Structures, 15: 805-827.
MindLIN, R. 1936. Force at a point in the interior of a semi-infinite solid. Physics, 7: 195-202.

MuKI, R. 1960. Asymmetric problem of the theory of elasticity for a semi-infinite solid and a thick place. In Progress in solid mechanics. Vol. 1. Edited by I. N. Sneddon and R. Hill. North Holland, Amsterdam, The Netherlands. pp. 399-439.

MUk1, R., and Sternberg, E. 1969. On the diffusion of an axial load from an infinite cylindrical bar embedded in an elastic medium. International Journal of Solids and Structures, 5: 587-605.

- 1970. Elastostatic load transfer to a halfspace from a partially embedded axially loaded rod. International Journal of Solids and Structures, 6: 69-90.

Niumpradit, B., and Karasudhi, P. 1979. Load transfer from an elastic pile to a saturated porous elastic soil. International Journal for Numerical and Analytical Methods in Geomechanics, 5: $115-138$.

Poulos, H. G., and Davis, E. H. 1968. The settlement behaviour of a single axially loaded incompressible piles and piers. Geotechnique, 18: $351-371$.

Selvadural, A. P. S., and Rajapakse, R. K. N. D. 1985. On the load transfer from a rigid cylindrical inclusion into an elastic halfspace. International Journal of Solids and Structures, 21: 1213-1229.

1987. A variational scheme for the analysis of torsion of nonuniform elastic bars embedded in elastic media. ASCE Journal of Engineering Mechanics, 113: 1534-1550.

SuriYamongkol, S., Karasudhi, P., and Lee, S. L. 1973. Axially loaded rigid cylindrical body embedded in an elastic halfspace. Proceedings, 13th Midwestern Mechanics Conference, Pittsburgh, PA, pp. $333-347$. 